1 The Phlebotomus papatasi transcriptomic response to trypanosomatid-contaminated blood is robust but non-specific

3

4

5

6

7 Megan A. Sloan ${ }^{1}$, Jovana Sadlova ${ }^{3}$, Tereza Lestinova ${ }^{3}$, Mandy J. Sanders², James A. Cotton², Petr Volf ${ }^{3}$, Petros Ligoxygakis ${ }^{1^{*}}$

9

10

1. Department of Biochemistry, University of Oxford, South Parks Rd OX1 3QU Oxford UK.

2. The Wellcome Sanger Institute, Wellcome Genome Campus, Hinxton, Cambridgeshire CB10 1SA UK. Republic. 
Abstract

25 Leishmaniasis, caused by parasites of the genus Leishmania, is a disease that effects up to 8

26 million people worldwide. Parasites are transmitted to human and animal hosts through the

27 bite of an infected sand fly. Novel strategies for disease control, require a better

28 understanding of the key step for transmission namely, the establishment of infection inside

29 the fly. In this work we wanted to identify fly transcriptomic signatures associated with

30 infection success or failure. We used next generation sequencing to describe the

31 transcriptome of the sand fly Phlebotomus papatasi when fed with blood alone or with blood

32 containing one of three trypanosomatids: Leishmania major, Leishmania donovani and

33 Herpetomonas muscarum: a parasite not transmitted to humans. Of these, only L. major was

34 able to successfully establish an infection in P. papatasi. However, the transcriptional

35 signatures observed were not specific to success or failure of infection but a generalised

36 response to the blood meal. This implies that sand flies perceive Leishmania as just a feature

37 of their microbiome landscape and that any strategy to tackle transmission should focus on

38 the response towards the blood meal rather than parasite establishment.

39

40 


\section{Authors summary}

42 Leishmania are parasites that cause leishmaniasis, a group of serious diseases that affect

43 millions of people, mainly across the subtropics and tropics. They are transmitted to humans

44 by phlebotomine sand flies. However, despite establishment in the insect's midgut being key

45 to transmission, early infection events inside the insect are still unclear. Here, we study the gene expression response of the insect vector to a Leishmania parasite that is able to establish

47 infection (L. major) one that is unable to do so (L. donovani) as well as one that is not a natural parasite of sand flies (Herpetomonas muscarum). We found that responses following any of the infected blood meals was very similar to uninfected blood meal. However, changes post-

50 blood meal from day 1 to day 9 were dramatic. As a blood feeding insect can accumulate

51 three times its weight in one blood meal, this seems to be the most important physiological

52 change rather than the presence of the parasite. The latter might be just one in a number of

53 microbes the insect encounters. This result will generate new thinking around the concept of

54 stopping transmission by controlling the parasite inside the insect. 
57 Leishmaniasis, a disease caused by parasites of the genus Leishmania, is endemic in 85 territories across the globe - with up to 8 million people affected ${ }^{1}$. Parasites infect vertebrates through the bite of an infected sand fly vector (Diptera: Phlebotominae). The acute form of disease, visceral leishmaniasis (VL) or kala-azar, is fatal in $95 \%$ of untreated cases and claims up to 50 thousand lives annually - though non-fatal infections causing dermatological symptoms are most common ${ }^{1}$. The ongoing $\mathrm{VL}$ elimination program in the Indian subcontinent is proving successful against the most severe clinical forms of $\mathrm{VL}^{2}$. However, elimination of leishmaniasis will likely require a combination of transmission blocking strategies and novel treatments. This is especially the case in light of reports of resistance to drugs used to treat human infections ${ }^{3,4}$, as well as pesticides used to control vector populations $^{5-7}$. But to develop approaches to blocking transmission, we need a better understanding of the basic biology that underlines the interactions between parasite and insect vector.

The sand fly responses to blood feeding have been investigated with several gene families shown to be transcribed and/or expressed in response to a blood meal ${ }^{8}$. These include: digestive enzymes such as trypsins and chymotrypsins, pathogen recognition molecules and components of the peritrophic matrix - a protective chitinous mesh which lines the midgut after ingestion ${ }^{8}$. However, few sand fly genes or transcripts specifically associated with Leishmania infection. There is some evidence to suggest Leishmania are able to modify host responses to promote survival and infection establishment. Analysis of cDNAs

77 isolated from dissected Phlebotomus papatasi $i^{9}$ and Phlebotomus perniciosus ${ }^{10}$ midguts revealed that several transcripts which are enriched after receipt of a blood meal are depleted when flies are fed blood containing Leishmania. These included digestive proteases, such as 
80

trypsins, as well as peritrophins which are chitin-binding components of the peritrophic matrix - a protective chitinous mesh which lines the midgut after ingestion ${ }^{11}$ and serves as a temporary barrier to leishmania ${ }^{12}$.

Recently, we described both the host ${ }^{13}$ and parasite ${ }^{14}$ transcriptomes in another trypanosomatid-insect infection model namely, Drosophila melanogaster and its own natural trypanosomatid parasite Herpetomonas muscarum. We showed that parasite feeding resulted in differential transcription of the two NF-KB pathways Toll and Imd, the dual oxidase pathway and STAT-dependant epithelial stem cell proliferation. Additionally, we found ${ }^{14}$ that the $H$. muscarum transcriptome during infection closely resembled that reported for L. major during Phlebotomus duboscqi infection ${ }^{15}$.

Given this, we wished to compare the Drosophila responses to those of sand flies during infection. Common transcriptomic signatures between the two systems would indicate an evolutionarily conserved response to trypanosomatid infection. Such responses would be of great interest for the development of broad-spectrum transmission blocking strategies for trypanosomatid diseases. However, no comparably comprehensive data' is available from sand flies. As such we sought to describe the sand fly transcriptomic responses to trypanosomatid infection using next generation sequencing techniques (RNA-seq). Herein, we describe the transcriptome of $P$. papatasi at three timepoints corresponding to important stages of trypanosomatid infection; 1 day post blood meal (PBM); following blood meal digestion and when parasites can be found attached to the midgut epithelium (4 days PBM); when parasites have migrated anteriorly in the gut and are found in the thoracic midgut and the stomodeal valve of the fly (9 days PBM, Figure 1$)^{16}$. Infections were done in the context of both permissive (Leishmania major) and refractory (Leishmania donovani) infections, as well as the with monoxenous (infects only insects) trypanosomatid $H$. muscarum, which is not 
a natural parasite of sand flies. Using this strategy, we hoped to identify host transcriptional signatures associated with permissive and refractory infection outcomes - in addition to identifying evolutionarily conserved host responses as described above. feeding is robust and extensive, with the differentially regulation of thousands of genes -

110 (in blood) fed flies. Blood alone appears to trigger transcription of genes from several immune pathways - including Imd, Toll and JAK-STAT. Activation of these responses despite the absence of parasites in the meal may be a pro-active strategy by the sand flies to prevent infection.

\section{Read mapping, de novo transcript assembly and differential expression analysis}

118 RNA was purified from sand flies at 1, 4 and 9 days PBM and the resulting reads sequenced

119 and mapped against the $P$. papatasi genome (Ppapl1, vectorbase ${ }^{17}$ ). The number of reads 120 generated per sample ranged from 1.08-12.05 million reads with 69.7-79.3\% mapping to the

121 P. papatasi genome in each sample (Table S1). Upon visual inspection of read mapping using

122 IGV $^{18}$ it appeared that over $20 \%$ of reads were mapping to regions which lacked annotated

123 features. To include these potentially novel genes in our analysis we assembled de novo 124 concordantly mapped read pairs (from all samples) into 16,025 transcripts. The assembled 125 transcripts were then merged with the existing annotation of 11,834 transcripts, to give a 126 final set of 18,592 unique transcripts (see Supplementary data files). This represents 127 approximately $97.2 \mathrm{Mb}$ of $P$. papatasi transcriptome with an average transcript length of 
4,190 bp. All reads were then counted against the final set of transcripts for differential expression analysis. from days 4 and 9 PBM appearing similar. We also note that samples do not clearly group in accordance with trypanosomatid feeding status.

\section{Differential expression associated with trypanosomatid presence in the bloodmeal} difference in transcript abundances between blood-fed and L. major fed flies at any time point. Furthermore, we find in excess of twelve thousand genes for which we reject the comparisons (Wald test) between blood only fed and trypanosomatid fed flies (Table 1). muscarum and L. donovani feeding compared to blood only fed control flies. There were significantly fewer transcripts for the gene PPAI009043, an orthologue to the D. melanogaster signalling protein Rho GTPase activating protein at 54D (RhoGAP54D), in flies fed $H$.

147 Aedes aegypti and Anopheles gambiae RhoGAP54D orthologues are upregulated in blood fed mosquitos compared to sugar fed controls ${ }^{19,20}$. Given this, and that this response was not seen after Leishmania feeding, this transcriptomic response may be $H$. muscarum specific. 
unclear, however the protein is linked to epithelial morphogenesis during Drosophila

152

154

155

156

157

158

159

160

161

162

163

164

165

166

167

development ${ }^{21}$ and so may also play a role in the mature insect gut.

In L. donovani fed flies there were significantly fewer transcripts for the putative transporter TrpA1 (PPAI004036, log2foldchange 2.8, padj < 0.05) versus blood only fed flies at 9 days PBM. TrpA1 is more generally associated chemo- and thermo-sensing ${ }^{22,23}$ in Drosophila, however a study by Du et al. 2006 links TrpA1 to the expulsion of food-borne pathogens by increased defecation and the DUOX pathway (discussed further below) ${ }^{24}$. Speculatively, reduction in $\operatorname{TrpA} 1$ transcripts after $L$. donovani feeding may hint at modification of host defensive pathways to promote survival. We also find significantly more CUFF.12679 transcripts (log2-foldchange 16.8, p-adj < 0.05) in L. donovani fed flies. This novel transcript lacks conserved domains or sequence similarity to known Dipteran gene transcripts.

\begin{tabular}{|c|c|c|c|c|c|c|c|c|c|}
\hline \multirow{3}{*}{ vs. } & \multicolumn{9}{|c|}{$\begin{array}{c}\text { Number of genes which do not significantly change in expression by } 2 \text {-fold } \\
\text { or more in either direction }(p<0.05)\end{array}$} \\
\hline & \multicolumn{3}{|c|}{$\begin{array}{l}\text { Leishmania major fed } \\
\text { flies }\end{array}$} & \multicolumn{3}{|c|}{$\begin{array}{l}\text { Leishmania donovani } \\
\text { fed flies }\end{array}$} & \multicolumn{3}{|c|}{$\begin{array}{c}\text { Herpetomonas } \\
\text { muscarum fed flies }\end{array}$} \\
\hline & $\begin{array}{l}1 \text { day } \\
\text { PBM }\end{array}$ & $\begin{array}{c}4 \\
\text { days } \\
\text { PBM }\end{array}$ & $\begin{array}{l}9 \text { days } \\
\text { PBM }\end{array}$ & $\begin{array}{l}1 \text { day } \\
\text { PBM }\end{array}$ & $\begin{array}{c}4 \\
\text { days } \\
\text { PBM }\end{array}$ & $\begin{array}{c}9 \\
\text { days } \\
\text { PBM }\end{array}$ & $\begin{array}{l}1 \text { day } \\
\text { PBM }\end{array}$ & $\begin{array}{c}4 \\
\text { days } \\
\text { PBM }\end{array}$ & $\begin{array}{c}9 \\
\text { days } \\
\text { PBM }\end{array}$ \\
\hline $\begin{array}{l}\text { Blood fed } 1 \\
\text { day PBM }\end{array}$ & 12586 & & & 12797 & & & 11957 & & \\
\hline $\begin{array}{l}\text { Blood fed } 4 \\
\text { days PBM }\end{array}$ & & 12188 & & & 12597 & & & 12356 & \\
\hline $\begin{array}{l}\text { Blood fed } 9 \\
\text { days PBM }\end{array}$ & & & 12762 & & & 12731 & & & 12634 \\
\hline
\end{tabular}

Table 1 - Numbers of transcripts which do not significantly change in expression by 2-fold or more in either direction between blood fed and trypanosomatid fed P. papatasi $(p<0.05)$. 
Direct comparisons between trypanosomatid infections yielded similarly few differentially expressed transcripts (Table S3). At day 1 PBM the only differentially expressed transcript between the three infections was that of trypsin 1 (PPAI010956, padj < 0.05) which was 2-fold enriched in $H$. muscarum fed flies compared to those fed L. donovani.

174 the ectoperitrophic space persist to develop mature infection ${ }^{25}$. Despite the differences in the 175 infection outcome reported in laboratory infections across the three trypanosomatids ${ }^{16}$, 176 there were few differences in the host transcriptome at this critical time point. Two 177 transcripts were found to be significantly differentially abundant - one corresponding to the 178 PPAI000999 gene and the other a novel transcript CUFF.14170. Both transcripts were found 179 at significantly higher levels ( $p$-adjusted $<0.05$, log2foldchanges of 4 and 18 respectively) in H. muscarum fed flies compared to those fed L. donovani. PPAI000999 encodes a protein predicted to bind to chitin (GO:0006030, GO:0008061 and smart00494). The novel transcript CUFF.14170 has no known conserved domains and BLAST searches against Dipteran sequences did not yield any significant hits. transcripts were differentially expressed between Leishmania fed and $H$. muscarum fed flies

187 discussed TrpA1 (PPAI004036), and significantly less for the putative zinc metalloprotease PPAI010164 and novel transcript CUFF.12679, than those fed H. muscarum. Flies fed L. major had significantly more transcripts for the hypothetical protein PPAI002947. Additionally, 

(padj <0.05). of transcriptional variation in these flies and not trypanosomatid infection. As such we further investigated transcriptomic changes after blood feeding alone in P. papatasi.

\section{The Phlebotomus papatasi transcriptome after blood feeding}

Ingestion of blood alone was associated with significant changes to the transcriptome. The of the blood meal remnants the transcriptome was comparatively stable with 264 differentially regulated transcripts (4 vs. 9 days PBM, Table S5). Due to the large number of differentially expressed transcripts highlighted by these comparisons we first investigated transcripts whose log 2 fold change was $>4$ in either direction between timepoints. From this subset we were able to focus our analysis on a number of key genes and pathways which are discussed further below (Tables S6 and S7).

207 metabolism and immunity Of the 217 transcripts differentially regulated > 4-fold between 1 and 4 days PBM, 197 transcripts were found to be comparatively enriched at day 1 PBM and 20 were comparatively enriched at day 4 PBM. Ninety-eight of these transcripts did not contain known conserved domains. 

groups differentially regulated between day 1 and day 4 PBM. We observed upregulation of 9 transcripts for putative trypsins and chymotrypsins - including the previously characterised chymotrypsins 1 (PPAI010833), chymotrypsin 3 (PPAI005023) and trypsin 4

218 (PPAI010456) ${ }^{8,11,26}$. We also observed upregulation of transcripts which may represent novel trypsins, based on conserved domains and similarity to other Dipteran trypsin/chymotrypsin sequences, as they are not included in the current genome annotation (Ppap $v 1^{17}$ ) (CUFF.11666, CUFF.9493, CUFF.6542) and chymotrypsins (CUFF.15058, CUFF.16005, CUFF.15086, CUFF.14587, CUFF.12454). In contrast, the transcript putatively encoding for trypsin 1 (PPAI010956) was shown to be enriched at day 4 PBM compared to the earlier timepoint. The roles of trypsin and chymotrypsin-like serine proteases during blood digestion in hematophagous insects are well characterised with expression levels varying according to type and the time since the last blood meal. Our findings agree with previous work, which showed upregulation of trypsins $3 / 4$ and chymotrypsin 1 in response to the blood meal, as well as the decrease of trypsin $1^{26}$.

In addition to the trypsins themselves, five transcripts whose products are predicted to contain trypsin inhibitor like domains (PPAI003932, PPAI000270, PPAI000272, PPAI000274, PPAI003557) were also comparatively enriched at day 1 PBM (vs. day 4 PBM). It is possible the corresponding proteins play roles in the regulation of the trypsin 1 as well as other trypsins (e.g. trypsin 2), reported to be downregulated after blood feeding ${ }^{26}$. Several transcripts encoding for proteins with predicted serine protease/proteolytic activity, the sequences of which do not resemble trypsins/chymotrypsins were also comparatively enriched at day 1 PBM. These included two known genes (PPAI009419, 
proteases are implicated in several other cellular processes including innate immune signalling - notably in Toll pathway activation ${ }^{27}$ and the melanisation response ${ }^{28}$. The

240 predicted protein for PPAI009419 shares approx. 51\% identity with the Culex

241 quinquefasciatus CLIPA15 (also known as masquerade) across its sequence. CLIPA proteases

242 interact with and regulate other CLIPs, and the prophenoloxidases (PPO), involved in the

243 melanisation repsonse $\mathrm{e}^{29,30}$. This response produces reactive quinones which then polymerise

244 to form the dark insoluble pigment melanin. These molecules can encapsulate and isolate

245 invading pathogens or toxic compounds. They also locally generate high local levels of

246 cytotoxic reactive oxygen species and prevents gas diffusion, starving the invading pathogen

247 of oxygen. In addition to the putative CLIPA transcript, four pro-phenoloxidase transcripts are upregulated early infection (PPO1 - PPA1008831, PPAI010450; PPO2 - PPAI012836, PPAI012835). These zymogens are the rate limiting enzymes in the production of melanin. PPO1/2 and CLIPA15 were also upregulated immediately after blood feeding in Anopheles gambiae $^{20}$ - suggesting this is a conserved response to blood feeding in Dipterans. play vital roles in protection against invading pathogens - peritrophins. These core components of the peritrophic matrix (PM) have been shown to be a major barrier against infection establishment. Knockdown of Peritrophin 1 (Per1) in P. papatasi results in an approximately $40 \%$ increase in Leishmania major load at 48 hours after parasite ingestion ${ }^{31}$.

257 In our study, Per1 transcripts were highly enriched at day 1 PBM (vs. day 4 PBM) with log2 fold change of 9.96. Of the 32 annotated peritrophins in the P. papatasi genome, 14 were

259 found to be significantly differentially regulated between days 1 and 4 PBM (Table 2). The majority of transcripts were comparatively enriched at day 1 PBM, however Per2 and Per28

261 transcripts were more abundant at later timepoints. Ramalho-Ortigão et al. $2007^{10}$ showed 
that $P$. papatasi peritrophin 1 (Per1) transcripts were enriched in flies fed a blood meal compared to a sugar meal, whilst peritrophin 2 (Per2) transcripts were comparatively depleted in blood fed flies. Additionally, the group showed that transcripts for both Per1 and Per2 were depleted in L. major infected flies compared to those fed only blood ${ }^{11}$. Our data largely agree with these findings. However, transcript levels were not statistically significant different between trypanosomatid and blood-only fed flies - though we do observe fewer transcripts for Per2 (PPAI009723) in trypanosomatid-fed flies at day 4 PBM (Figure S1). Other than Per2, the patterns in peritrophin transcript abundance for trypanosomatid fed flies resembled those of the blood fed controls. resembles the Lutzomyia longipalpis protein ChiBi (EU124616.132, 84\% protein sequence identity). ChiBi was shown to be enriched in L. longipalpis fed with blood containing $L$. infantum chagasi ${ }^{32}$. Its upregulation here in P. papatasi in the absence of Leishmania may indicate this upregulation is a more general response to blood meal, rather than an infectionspecific response.

278 


\begin{tabular}{|c|c|c|c|}
\hline $\begin{array}{c}\text { Gene } \\
\text { name }\end{array}$ & Gene ID & $\begin{array}{c}\text { Log2fold } \\
\text { change }\end{array}$ & $\begin{array}{c}\text { p-value (Benjamini- } \\
\text { Hochberg adjusted) }\end{array}$ \\
\hline Per1 & PPAI009353 & 9.97 & $2.94 \mathrm{E}-30$ \\
\hline Per26 & PPAI004431 & 3.79 & $1.48 \mathrm{E}-02$ \\
\hline Per6 & PPAI001604 & 3.29 & $1.61 \mathrm{E}-07$ \\
\hline Per10 & PPAI004716 & 2.33 & $1.01 \mathrm{E}-04$ \\
\hline Per7 & PPAI002253 & 2.33 & $1.26 \mathrm{E}-03$ \\
\hline Per12 & PPAI001263 & 2.02 & $3.92 \mathrm{E}-02$ \\
\hline Per11 & PPAI004749 & 2.01 & $4.17 \mathrm{E}-11$ \\
\hline Per27 & PPAI008214 & 1.84 & $1.57 \mathrm{E}-02$ \\
\hline Per13 & PPAI004750 & 1.71 & $1.39 \mathrm{E}-02$ \\
\hline Per8 & PPAI002033 & 1.58 & $8.38 \mathrm{E}-11$ \\
\hline Per3 & PPAI006556 & 1.49 & $2.39 \mathrm{E}-06$ \\
\hline Per4 & PPAI006974 & 0.95 & $2.43 \mathrm{E}-02$ \\
\hline Per28 & PPAI001796 & -1.19 & $1.05 \mathrm{E}-03$ \\
\hline Per2 & PPAI009723 & -2.28 & $8.53 \mathrm{E}-07$ \\
\hline
\end{tabular}
metabolism associated genes were found to be upregulated at day 1 PBM. In addition, eight transcripts corresponded to known extracellular carboxylic ester hydrolases (PPAI002323, PPAI003061, PPAI003086, PPAI005115, PPAI005116, PPAI005680, PPAI009133, PPAI008993). membrane fatty acid desaturase genes (PPAI008098 and PPAI002108) were shown to be comparatively enriched at day 1 PBM. One transcript, CUFF.7417, does not correspond to a known gene, however the transcript showed strong sequence similarity to the extracellular 
identity). Additionally, CUFF.7417 is immediately downstream of PPAI005115/6 in the

301

302

303

304

305

306

307

308

309

310

311

312

313

314

315 not required after digestion. transmembrane domains.

genome and as such we propose this represents a previously unknown paralogue.

Four transcripts coded for proteins with solute carrier domains (cl00456). These transcripts encode for the two paralogous sodium-coupled monocarboxylate transporters (SCMTs, PPAI005125 and PPAI007402) and two putative SCMTs (CUFF.14648 and CUFF.14649). The SCMTs are transmembrane proteins, which move molecules with a single carboxylate group including pyruvate and lactate, across the plasma membrane in a protondependent manner and are associated with the insect midgut brush boarder ${ }^{33}$.

We found two transcripts, CUFF.17209 and CUFF.15972, whose products are predicted to contain the conserved insect allergen related repeat domain (pfam06757). These transcript sequences also showed similarity to reported cDNAs for P. papatasi microvillar proteins MVP1 and 2 respectively (>89\% identity to mRNA sequences). These proteins were also found previously to be upregulated in sand flies upon ingestion of a blood meal compared to sucrose-fed flies ${ }^{11}$. These transcripts could not be assigned to an annotated gene in the current vector base genome (Ppal1 $\left.{ }^{17}\right)$. The function of these proteins is not well understood though they appear to have a conserved signal peptide at the $\mathrm{n}$-terminus and lack

Finally, three olfactory (Or57 - PPAI013155, Or99- PPAI013290 and the putative protein PPAI002404) and a gustatory receptor orthologous to sweet taste receptors of Drosophila (Gr9 - PPAI010978), were upregulated at day 1 PBM compared to later timepoints. It is likely these sensory receptors were involved in sensing and acquisition of the blood meal and subsequent decreases in their transcript abundances, may indicate these sensors were 
The transcriptome after defecation of the blood meal is comparatively stable corresponded to two glutamate receptors (PPAI003634, PPAI008275), apoptosis inhibitor survivin (PPAI002284), two histone methyltransferases (PPAI005539, PPAI005538) and a mucin (PPAI009152). Mucins have been implicated in the interaction with Leishmania parasites. Given that several immunity-related transcripts (including peritrophins, mucins and melanization pathway genes) were upregulated, we postulated that upon blood meal ingestion a general immune response was triggered. As such we investigated the transcription of the members of the two major innate pathways after a bloodmeal: Toll and Immunodeficiency (Imd). Both pathways have been shown to play a role in the response to trypanosomatids $^{13,34-38}$. Furthermore, we also investigated members of the Dual-oxidase (DUOX) and JAK-STAT pathways, both of which were implicated in $D$. melanogaster- $H$. muscarum interaction ${ }^{13}$. Differential regulation statistics for these transcripts can be found in Table S8.

Blood ingestion alone is associated with increased innate immune gene transcription compared to days 4/9 PBM (fold change $>2$, p-adjusted $<0.05$, Figure $3 \mathrm{~A}$ ). An exception to this was the spätzle processing enzyme (SPE) the putative transcript for which is enriched in the latter two timepoints along with several intracellular Toll pathway components. These 
trypanosomatids. However, only flies fed with blood containing L. major or L. donovani promastigotes had significantly higher levels of transcripts encoding Toll pathway inhibitor Cactus at day-1 PBM compared to day 4 PBM (> 2-fold, p-adj < 0.05). Cactus transcript abundance was not significantly different between days 1 and 4 PBM in blood only or $H$. muscarum fed flies. peptidoglycan recognition proteins (PGRPs) were more prevalent at day 1 PBM compared to later timepoints (Figure 6B). However, only putative PGRP 2 (CUFF.5670) was found to be statistically significantly enriched (2.23-fold) at day 1 PBM (vs. day 4/9 PBM, p-adj < 0.05). The transcripts putatively encoding IMD, and several other proteins downstream of IMD in the pathway were found to be significantly enriched (padj < 0.05 ) at 4 and 9 days PBM (vs. day 1 PBM), including: DREDD, TAK1 and IKK $\beta$. We also observed significant enrichment of transcripts putatively encoding negative regulators of the IMD pathway Caspar, dUSP36, Trabid at days 4 and 9 PBM (>2 fold, $p$-adj < 0.05). Interestingly, the IMD transcription factor Relish was not significantly differentially regulated in blood-only fed flies, however flies fed blood containing L. major or L. donovani promastigotes showed enrichment of putative Relish transcripts at day 1 PBM compared to at 4 and 9 days PBM. As such, whilst there is overall upregulation of IMD pathway transcription with or without trypanosomatids in the blood meal, there may be important differences in the expression levels of the innate effectors the meal regulates when Leishmania are present. Both Toll and IMD result in the expression of a suite of antimicrobial peptides.

369 Transcripts for these immune effectors were not significantly differentially regulated after blood feeding alone. However, flies fed with blood containing L. major or L. donovani 371 promastigotes were found to have significantly more transcripts for the AMP defensin at day 

peptides did change after trypanosomatid feeding, e.g. we observe elevated transcript levels for cecropin and attacin in some trypanosomatid infections (Figure $3 \mathrm{C}$ ), overall these were not found to be statistically significant changes. the NADPH oxidase, dual-oxidase (DUOX), through interaction of IMD with MEKK1 ${ }^{39}$. This to microbes. We found that DUOX transcripts were significantly higher at days 4 and 9 PBM

Figure 6C) - with no significant difference in DUOX transcript abundance between 4 and 9 days PBM in any infection condition. Similarly, we see significant increases in transcripts for genes upstream of DUOX across infection conditions including: the transcription factor ATF2, p38 kinase and MEKK1. As such, induction of DUOX pathway transcription appears to be a generalised response to blood feeding rather than an infection-specific response.

The JAK-STAT pathway is also associated with the dipteran response to trypanosomatids

389 Finally, given the association between the JAK-STAT pathway (Figure 4B), dipteran gut morphology and immunity ${ }^{40}$, particularly in a trypanosomatid infection context ${ }^{13}$, we also investigate the transcription of key components of this pathway after blood feeding. We observed higher abundance of putative Upd1 transcripts at day 1 PBM compared to later timepoints, however this change was only show to be statistically significant for flies fed with blood and L. major where there was a 2.2-fold enrichment of putative Upd1 transcripts. 
enriched in flies in all infection conditions at the two later time points (vs. 1 day PBM, p-adj < 0.05). We also observed a modest enrichment of transcripts for cytokine Upd2 and the transmembrane receptor Domeless at days 4 and 9 PBM compared to earlier timepoints (fold changes 1.19 and 1.74 respectively, $p$-adj < 0.05). The transcription pattern for signalling protein hopscotch resembled that of Domeless, however these transcripts were only found to be statistically significantly enriched in trypanosomatid fed flies (padj < 0.05). Together these observations suggested an increase in JAK-STAT signalling a few days after a blood meal in P. papatasi. Further work to investigate if this signalling translates to changes in gut homeostasis, such as the increased stem cell proliferation observed in the DrosophilaHerpetomonas model, will be important. Currently however, as transcript abundance for STAT92E is enriched in blood only fed controls this response does not appear to be trypanosomatid specific.

\section{Concluding remarks}

411 alone, and the little variation between blood meals spiked with trypanosomatids that produce very different infections we speculate that aforementioned defensive responses are not infection specific. Such a strong response to the blood meal alone is not surprising given the additional stresses associated with the hematophagous habit ${ }^{41}$. The high-risk nutrient attainment method drives the insects to take large volumes of blood at each meal e.g. mosquitoes and tsetse flies expand up to 3 times their pre-meal size a blood meal ${ }^{41,42}-$

417 putting enormous mechanical strain on the tissues. In addition to the volume, the content of 418 their meal presents additional problems: excess water/ions ${ }^{43}$, toxic compounds ${ }^{44}$ and 
victim's blood temperature of blood-feeding arthropods can rapidly ( $<60$ seconds) increase

421 by over $10^{\circ} \mathrm{C}$ during their meal ${ }^{47,48}$. All of which must be overcome even in meals which do not contain parasites. Additionally, activation of immune pathways in absence of known infection may be a strategy to 'pre-emptively' protect the host against pathogens/toxic compounds which may be present in the newly ingested meal. Such anticipatory responses have been reported in other hematophagous insects including malaria vector Anopheles gambiae $^{49}$. overall gut bacterial diversity ${ }^{50}$ coupled to an increased abundance of aerobic bacteria ${ }^{46}$. We suppose that these changes may mask any effects from the presence of trypanosomatids. However, there was also no significant difference between day 9 PBM L. major and the other specificity of the $P$. papatasi response and implies that for the fly, L. major is just another feature in the microbiome landscape.

\section{Materials and methods}

Phlebotomus papatasi maintenance.

439 A laboratory colony of P. papatasi (originating from Turkey) was maintained in the insectary 440 of the Charles University in Prague under standard conditions (at $26^{\circ} \mathrm{C}$ fed on $50 \%$ sucrose, 441 humidity in the insectary $60-70 \%$ with a $14 \mathrm{~h}$ light/10 $\mathrm{h}$ dark photoperiod) as described 442 previously ${ }^{51}$. 
Trypanosomatid maintenance. foetal bovine serum (FBS, Gibco) supplemented with 1\% BME vitamins (Basal Medium Eagle, Sigma), $2 \%$ sterile urine, $250 \mathrm{\mu g} / \mathrm{ml}$ amikacin (Amikin, Bristol-Myers Squibb) at $23^{\circ} \mathrm{C}(\mathrm{L}$. donovani, L. major) or $28^{\circ} \mathrm{C}$ (H. muscarum).

450

451

\section{Phlebotomus papatasi infections.}

452

Leishmania and $H$. muscarum promastigotes from log-phase cultures (day 3-4 post inoculation) were resuspended in defibrinated and heat-inactivated rabbit blood (LabMediaServis) at concentration $1 \times 10^{6}$ promastigotes per $\mathrm{mL}$ which corresponds to 500 1000 promastigotes per $P$. papatasi female ${ }^{25}$. Sand fly females (5-9 days old) were infected by feeding through a chick-skin membrane (BIOPHARM, Czech Republic) on the suspension. Engorged sand flies were maintained in the same conditions as the colony. Females were dissected at days 1, 4 and 9 post bloodmeal (PBM).

\section{RNA extraction}

RNA was extracted from pools of 10 sand flies from each condition and timepoint. Whole flies added. The homogenate was mixed and incubated at $4^{\circ} \mathrm{C}$ for an hour. Debris was then spun down by centrifugation at 12, $000 \mathrm{xg}$ for 5 mins and the resulting supernatant transferred to a new tube. To each sample $100 \mu \mathrm{L}$ of chloroform was added and samples incubated on ice 
were separated by centrifugation at $12,000 \mathrm{xg}$ for $15 \mathrm{~min}$ at $4{ }^{\circ} \mathrm{C}$ and the upper phase containing the RNA was moved to a new tube. Following this RNA extraction proceeded according to the TRIzol Reagent manufacturers protocol for RNA isolation. This protocol

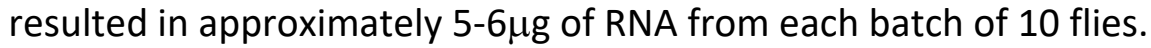

471

\section{Transcriptomic libraries}

473 Poly-A mRNA was purified from total RNA using oligodT magnetic beads and strand-specific

474 indexed libraries were prepared using the KAPA Stranded RNA-Seq kit followed by ten cycles

475 of amplification using KAPA HiFi DNA polymerase (KAPA Biosystems). Libraries were quantified and pooled based on a post-PCR Agilent Bioanalyzer and 75 bp paired-end reads were generated on the Illumina HiSeq v4 following the manufacturer's standard sequencing protocols. All raw sequencing reads are available (from the date of pending journal publication) on the European Nucleotide Archive under study accession number PRJEB35592.

\section{Read mapping and differential expression analysis.}

483 Reads were mapped to $P$. papatasi genome (Ppapl1 v1, Vectorbase ${ }^{17}$ ) using hisat2 ${ }^{52}$. Reads which mapped uniquely and in their proper pair were extracted and used to assemble transcripts de novo with Cufflinks (Tuxedo suite) $)^{53}$. The newly assembled transcripts were combined with the VectorBase transcript assembly to create a new set of transcripts using are included in the supplementary data files. Reads were then counted against the cufflinks- 
490

491

492

493

494

495

496

497

498

499

500

501

502

503

504

505

506

507

508

509

510

511

512

replicates for each sample were collapsed prior importing into $\mathrm{R}$ for differential expression analysis (pairwise Wald tests) in DESeq $2^{55}$.

Acknowledgements We thank the staff of the DNA pipelines at Wellcome Sanger Institute for sequencing and generating sequencing libraries. This work was supported by the European Commission, Horizon 2020 Infrastructure Infravec2 project (grant agreement No 731060, https://infravec2.eu). JS and PV were supported by ERD Funds, project CePaViP (CZ.02.1.01/16_019/0000759). MJS and JAC were supported by Wellcome via their core support for the Wellcome Sanger Institute (WSI) through grant 206194. Work in Oxford was supported by a Consolidator grant from the European Research Council (310912 DrosoParasite, to $\mathrm{PL}$ ), project grant $\mathrm{BB} / \mathrm{K003569}$ from the BBSRC (to $\mathrm{PL}$ ) and a Wellcome Trust doctoral scholarship (to MAS).

\section{References}

1. Courtenay, O., Peters, N. C., Rogers, M. E. \& Bern, C. Combining epidemiology with basic biology of sand flies, parasites, and hosts to inform leishmaniasis transmission dynamics and control. PLOS Pathog. 13, e1006571 (2017).

2. Sundar, S., Singh, O. P. \& Chakravarty, J. Visceral leishmaniasis elimination targets in India, strategies for preventing resurgence. Expert Rev. Anti. Infect. Ther. 16, 805-812 (2018).

3. Ponte-Sucre, A. et al. Drug resistance and treatment failure in leishmaniasis: A 21st century challenge. PLoS Negl. Trop. Dis. 11, (2017).

4. Basselin, M., Denise, H., Coombs, G. H. \& Barrett, M. P. Resistance to pentamidine in 
Leishmania mexicana involves exclusion of the drug from the mitochondrion.

Antimicrob. Agents Chemother. 46, 3731-3738 (2002).

515

5. Hassan, M. M. et al. Insecticide resistance in the sand fly, Phlebotomus papatasi from

6. Dhiman, R. C. \& Yadav, R. S. Insecticide resistance in phlebotomine sandflies in (2016).

7. Fawaz, E. Y. et al. Pyrethroid insecticide resistance mechanisms in the adult phlebotomus papatasi (diptera: Psychodidae). J. Med. Entomol. 53, 620-628 (2016).

8. Abrudan, J. et al. The characterization of the Phlebotomus papatasi transcriptome. Insect Mol. Biol. 22, 211-232 (2013).

9. Dillon, R. J. \& Lane, R. P. Influence of Leishmania infection on blood-meal digestion in the sandflies Phlebotomus papatasi and P. langeroni. Parasitol. Res. 79, 492-496 (1993).

10. Dostálová, A. et al. The midgut transcriptome of Phlebotomus (Larroussius) perniciosus, a vector of Leishmania infantum: comparison of sugar fed and blood fed sand flies. BMC Genomics 12, 223 (2011).

11. Ramalho-Ortigão, M. et al. Exploring the midgut transcriptome of Phlebotomus papatasi: comparative analysis of expression profiles of sugar-fed, blood-fed and Leishmania-major-infected sandflies. BMC Genomics 8, 300 (2007).

12. Sádlová, J. \& Volf, P. Peritrophic matrix of Phlebotomus duboscqi and its kinetics during Leishmania major development. Cell Tissue Res. 337, 313 (2009). 
PLOS Genet. 15, e1007931 (2019).

538 14. Sloan, M. A. et al. Transcriptional and genomic parallels between the monoxenous parasite Herpetomonas muscarum and Leishmania. PLOS Genet. 15, e1008452 (2019).

15. Inbar, E. et al. The Transcriptome of Leishmania major Developmental Stages in Their Natural Sand Fly Vector. mBio 8, (2017).

16. Dostálová, A. \& Volf, P. Leishmania development in sand flies: parasite-vector interactions overview. Parasit. Vectors 5, 276 (2012).

17. Giraldo-Calderón, G. I. et al. VectorBase: an updated bioinformatics resource for invertebrate vectors and other organisms related with human diseases. Nucleic Acids Res. 43, D707-D713 (2015).

18. Robinson, J. T. et al. Integrative genomics viewer. Nat. Biotechnol. 29, 24-26 (2011).

19. Bonizzoni, M. et al. RNA-seq analyses of blood-induced changes in gene expression in the mosquito vector species, Aedes aegypti. BMC Genomics 12, 82 (2011).

20. Marinotti, O. et al. Genome-wide analysis of gene expression in adult Anopheles gambiae. Insect Mol. Biol. 15, 1-12 (2006).

21. Greenberg, L. \& Hatini, V. Systematic expression and loss-of-function analysis defines spatially restricted requirements for Drosophila RhoGEFs and RhoGAPs in leg morphogenesis. Mech. Dev. 128, 5-17 (2011).

22. Kim, S. H. et al. \&lt;em\&gt;Drosophila\&lt;/em\&gt; TRPA1 channel mediates chemical avoidance in gustatory receptor neurons. Proc. Natl. Acad. Sci. 107, 8440 LP-8445 (2010).

23. Hamada, F. N. et al. An internal thermal sensor controlling temperature preference in 
561

562

563

564

565

566

567

568

569

570

571

572

573

574

575

576

577

578

579

580

581

582

583

584

24. Du, E. J. et al. TrpA1 Regulates Defecation of Food-Borne Pathogens under the Control of the Duox Pathway. PLOS Genet. 12, e1005773 (2016).

25. Pruzinova, K. et al. Comparison of Bloodmeal Digestion and the Peritrophic Matrix in Four Sand Fly Species Differing in Susceptibility to Leishmania donovani. PLoS One 10, e0128203 (2015).

26. Ramalho-Ortigao, M., Kamhawi, S., Rowton, E., Ribeiro, J. M. C. \& Valenzuela, J. Cloning and characterization of trypsin and chymotrypsin-like proteases from the midgut of the sandfly vector Phlebotomus papatasi. Insect Biochem. Mol. Biol. 33, 163-171 (2003).

27. Buchon, N. et al. A single modular serine protease integrates signals from patternrecognition receptors upstream of the Drosophila Toll pathway. Proc. Natl. Acad. Sci. $106,12442-12447$ (2009).

28. An, C., Zhang, M., Chu, Y. \& Zhao, Z. Serine protease MP2 activates prophenoloxidase in the melanization immune response of Drosophila melanogaster. PLoS One 8, e79533-e79533 (2013).

29. Yassine, H., Kamareddine, L., Chamat, S., Christophides, G. K. \& Osta, M. A. A serine protease homolog negatively regulates TEP1 consumption in systemic infections of the malaria vector Anopheles gambiae. J. Innate Immun. 6, 806-818 (2014).

30. Kan, H. et al. Molecular Control of Phenoloxidase-induced Melanin Synthesis in an Insect. J. Biol. Chem. 283, 25316-25323 (2008).

31. Coutinho-Abreu, I. V, Sharma, N. K., Robles-Murguia, M. \& Ramalho-Ortigao, M. Characterization of Phlebotomus papatasi Peritrophins, and the Role of PpPer1 in Leishmania major Survival in its Natural Vector. PLoS Negl. Trop. Dis. 7, e2132 (2013).

32. Jochim, R. C. et al. The midgut transcriptome of Lutzomyia longipalpis: comparative 
analysis of cDNA libraries from sugar-fed, blood-fed, post-digested and Leishmania infantum chagasi-infected sand flies. BMC Genomics 9, 15 (2008).

33. Javed, M. A., Coutu, C., Theilmann, D. A., Erlandson, M. A. \& Hegedus, D. D. vesicles. Insect Sci. 26, 424-440 (2019).

34. Telleria, E. L. et al. Caspar-like Gene Depletion Reduces Leishmania Infection in Sand Fly Host Lutzomyia longipalpis. J. Biol. Chem. 287, 12985-12993 (2012).

35. Tinoco-Nunes, B. et al. The sandfly Lutzomyia longipalpis LL5 embryonic cell line has active Toll and Imd pathways and shows immune responses to bacteria, yeast and Leishmania. Parasit. Vectors 9, 222 (2016).

36. Boulanger, N., Bulet, P. \& Lowenberger, C. Antimicrobial peptides in the interactions between insects and flagellate parasites. Trends Parasitol. 22, 262-268 (2006).

37. Boulanger, N. et al. Characterization of a defensin from the sand fly Phlebotomus duboscqi induced by challenge with bacteria or the protozoan parasite Leishmania major. Infect. Immun. 72, 7140-7146 (2004).

38. Hu, C. \& Aksoy, S. Innate immune responses regulate trypanosome parasite infection of the tsetse fly Glossina morsitans morsitans. Mol. Microbiol. 60, 1194-1204 (2006).

39. Ha, E.-M. et al. Regulation of DUOX by the Gaq-phospholipase $\mathrm{C} \beta-\mathrm{Ca} 2+$ pathway in

40. Myllymaki, H. \& Ramet, M. JAK/STAT pathway in Drosophila immunity. Scand. J. Immunol. 79, 377-385 (2014).

41. Benoit, J. B. \& Denlinger, D. L. Bugs battle stress from hot blood. Elife 6, e33035 (2017). 
(Cambridge University Press, 2005).

610

611

612

613

614

615

616

617

618

619

620

621

622

623

624

625

626

627

628

629

630

631

632

43. Beyenbach, K. W. \& Piermarini, P. M. Transcellular and paracellular pathways of transepithelial fluid secretion in Malpighian (renal) tubules of the yellow fever mosquito Aedes aegypti. Acta Physiol. 202, 387-407 (2011).

44. Sterkel, M., Oliveira, J. H. M., Bottino-Rojas, V., Paiva-Silva, G. O. \& Oliveira, P. L. The dose makes the poison: nutritional overload determines the life traits of bloodfeeding arthropods. Trends Parasitol. 33, 633-644 (2017).

45. Wang, Y., Gilbreath III, T. M., Kukutla, P., Yan, G. \& Xu, J. Dynamic gut microbiome across life history of the malaria mosquito Anopheles gambiae in Kenya. PLoS One 6, e24767 (2011)

46. Volf, P., Kiewegova, A. \& Nemec, A. Bacterial colonisation in the gut of Phlebotomus duboseqi (Diptera: Psychodidae): transtadial passage and the role of female diet. Folia Parasitol. (Praha). 49, 73-77 (2002).

47. Lahondere, C. et al. Countercurrent heat exchange and thermoregulation during blood-feeding in kissing bugs. Elife 6, e26107 (2017).

48. Lahondère, C. \& Lazzari, C. R. Thermal effect of blood feeding in the telmophagous fly Glossina morsitans morsitans. J. Therm. Biol. 48, 45-50 (2015).

49. Upton, L. M., Povelones, M. \& Christophides, G. K. Anopheles gambiae blood feeding initiates an anticipatory defense response to Plasmodium berghei. J. Innate Immun. 7, 74-86 (2015).

50. Kelly, P. H. et al. The Gut Microbiome of the Vector \&lt;em\&gt; Lutzomyia longipalpis\&lt;/em\&gt; Is Essential for Survival of \&lt;em\&gt;Leishmania infantum\&lt;/em\&gt; MBio 8, e01121-16 (2017).

51. Volf, P. \& Volfova, V. Establishment and maintenance of sand fly colonies. J. Vector 
634 52. Kim, D., Langmead, B. \& Salzberg, S. L. HISAT: a fast spliced aligner with low memory requirements. Nat. Methods 12, 357 (2015).

636

53. Trapnell, C. et al. Differential gene and transcript expression analysis of RNA-seq experiments with TopHat and Cufflinks. Nat. Protoc. 7, 562 (2012).

54. Liao, Y., Smyth, G. K. \& Shi, W. featureCounts: an efficient general purpose program for assigning sequence reads to genomic features. Bioinformatics 30, 923-930

640 (2014).

641 55. Love, M. I., Huber, W. \& Anders, S. Moderated estimation of fold change and 


\section{Figures}

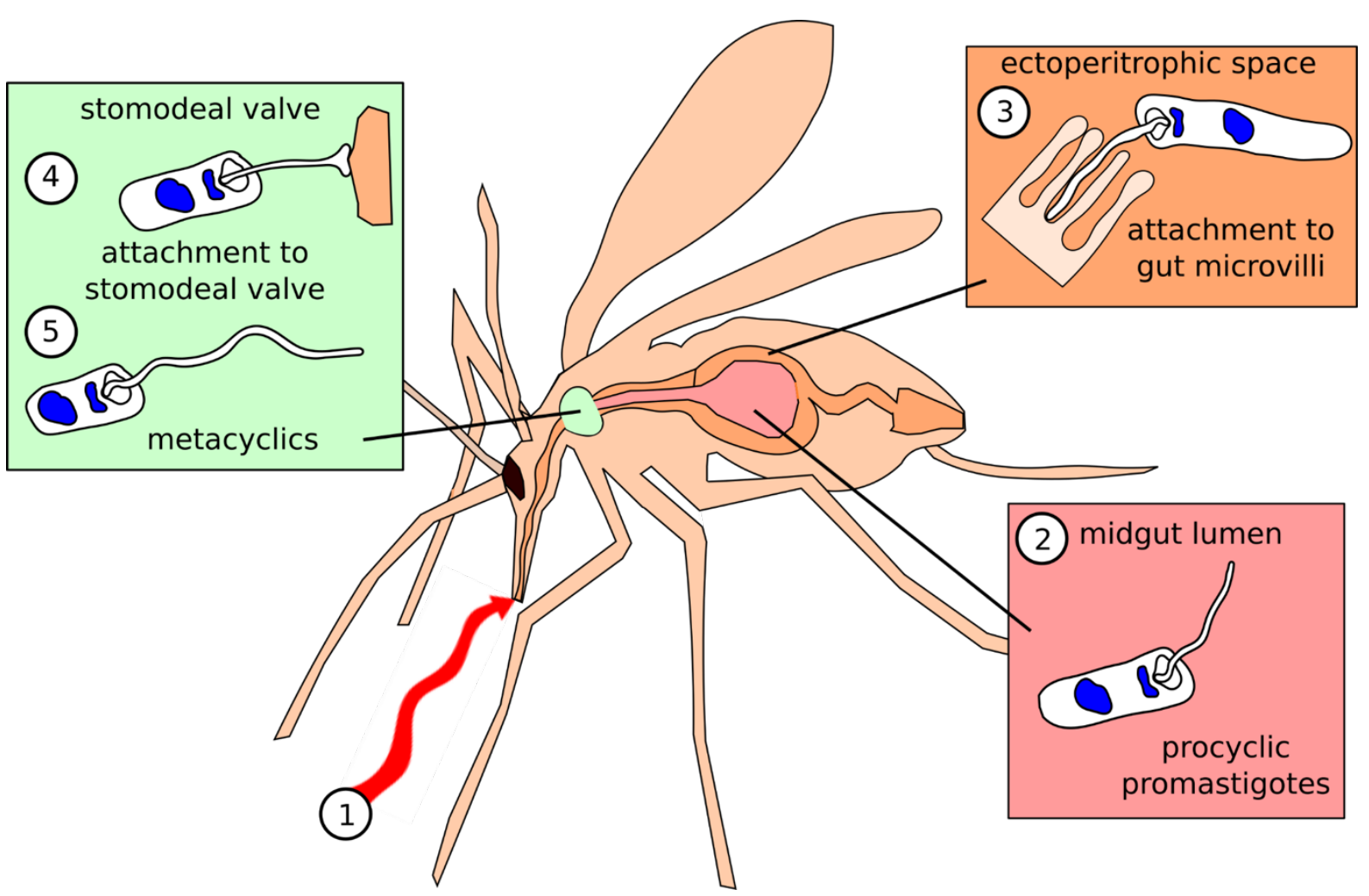

659

Figure 1 - Schematic of the 3 major Leishmania stages in sand flies. Shortly after ingestion

661 (1) of the blood meal promastigotes are localized in the midgut lumen, in the bloodmeal until the PM is broken at the end of digestion, they enter the endoperitrophic space and attach to the epithelial wall (3) (> 4 days PBM). Finally, where parasites have migrated anteriorly to the thoracic midgut and the stomodeal valve of the fly and the human-infective metacyclic forms differentiate from the earlier stages $(\mathbf{4 / 5},>9$ days PBM). 


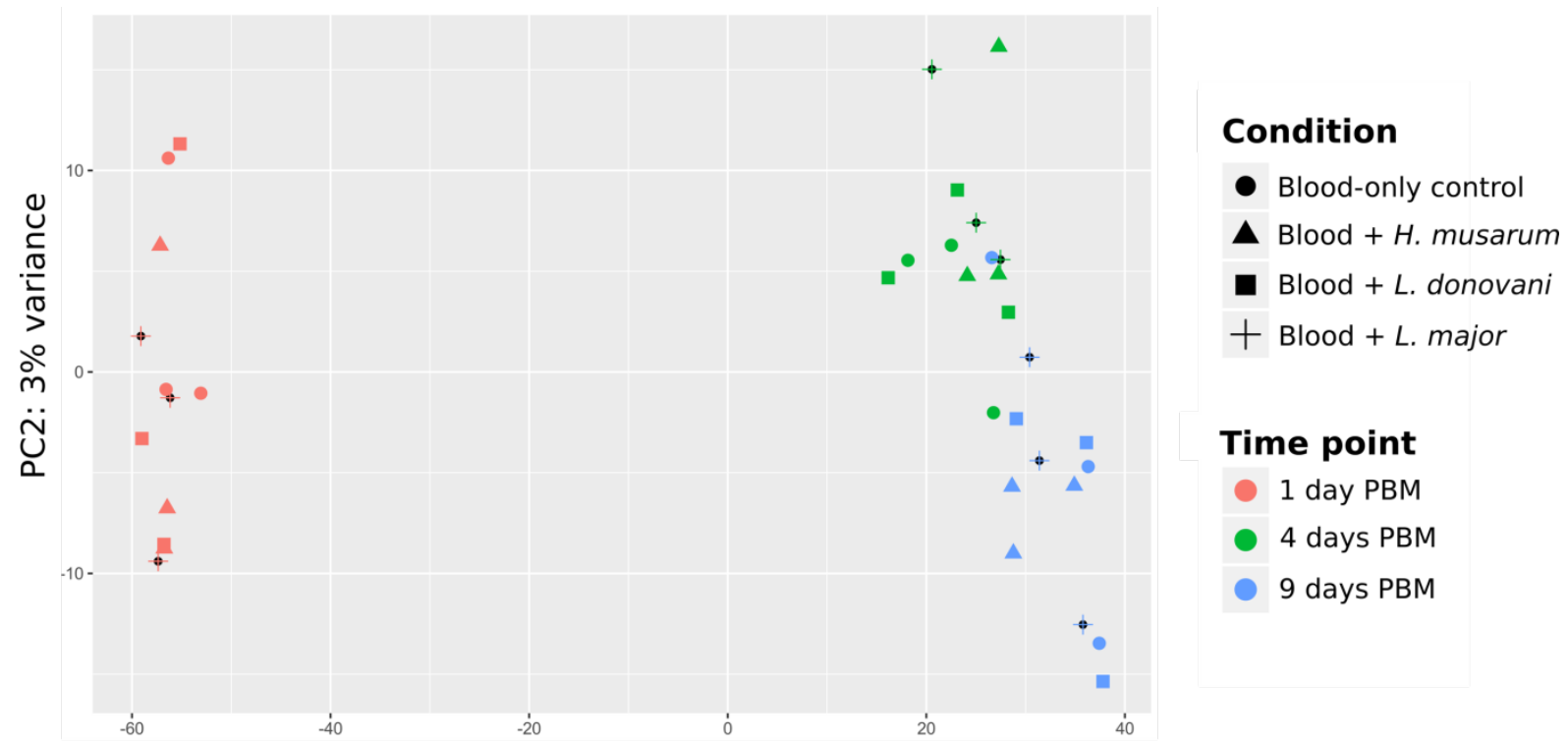

671 Figure 2-Principal component analysis shows that time was the major source of

672 variation, not infection status (condition).

673

674

675

676

677

678

679

680

681

682 

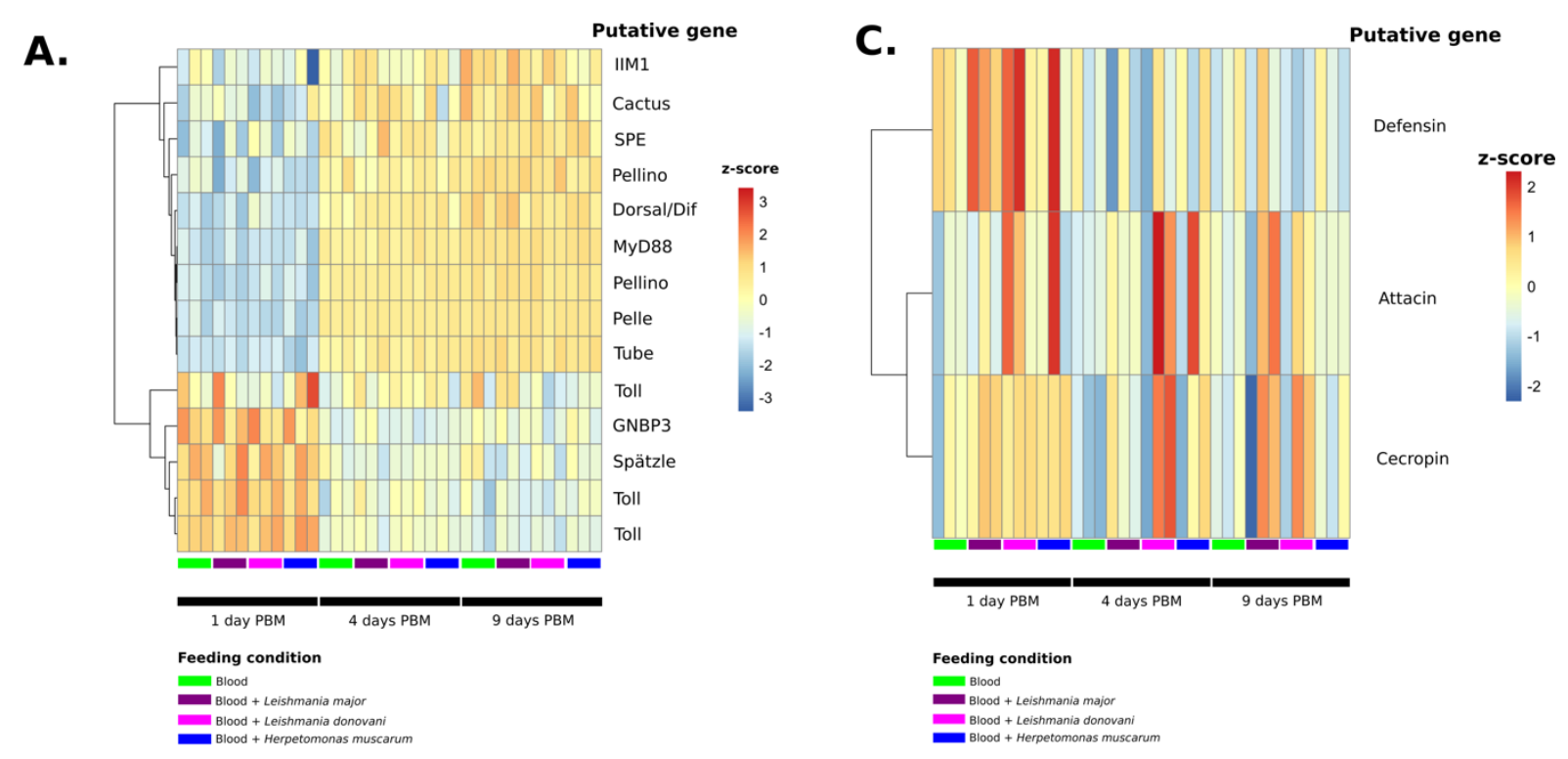

B.

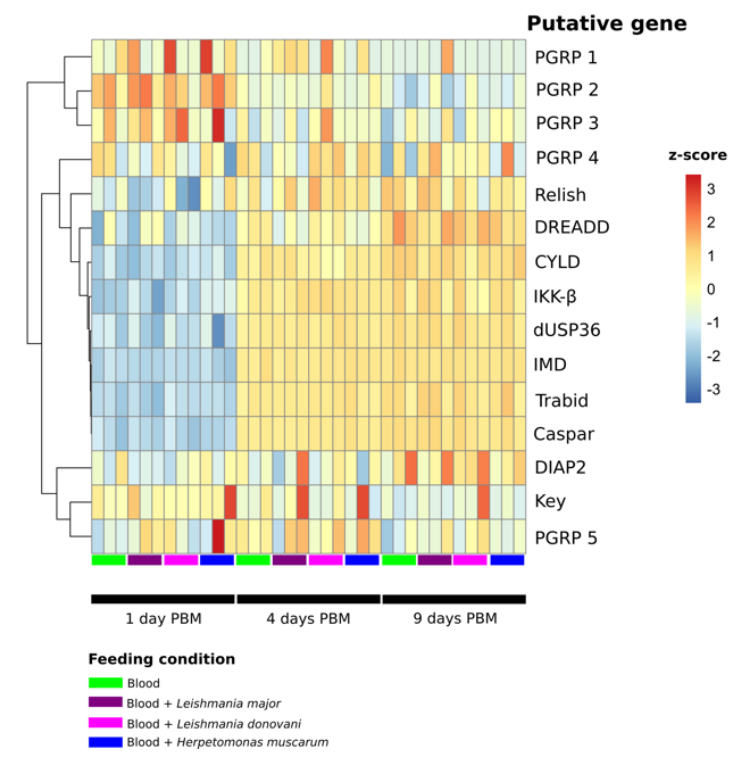

684

Figure 3 - Transcription of genes form the two major innate immune pathways in $P$. genes across samples. 


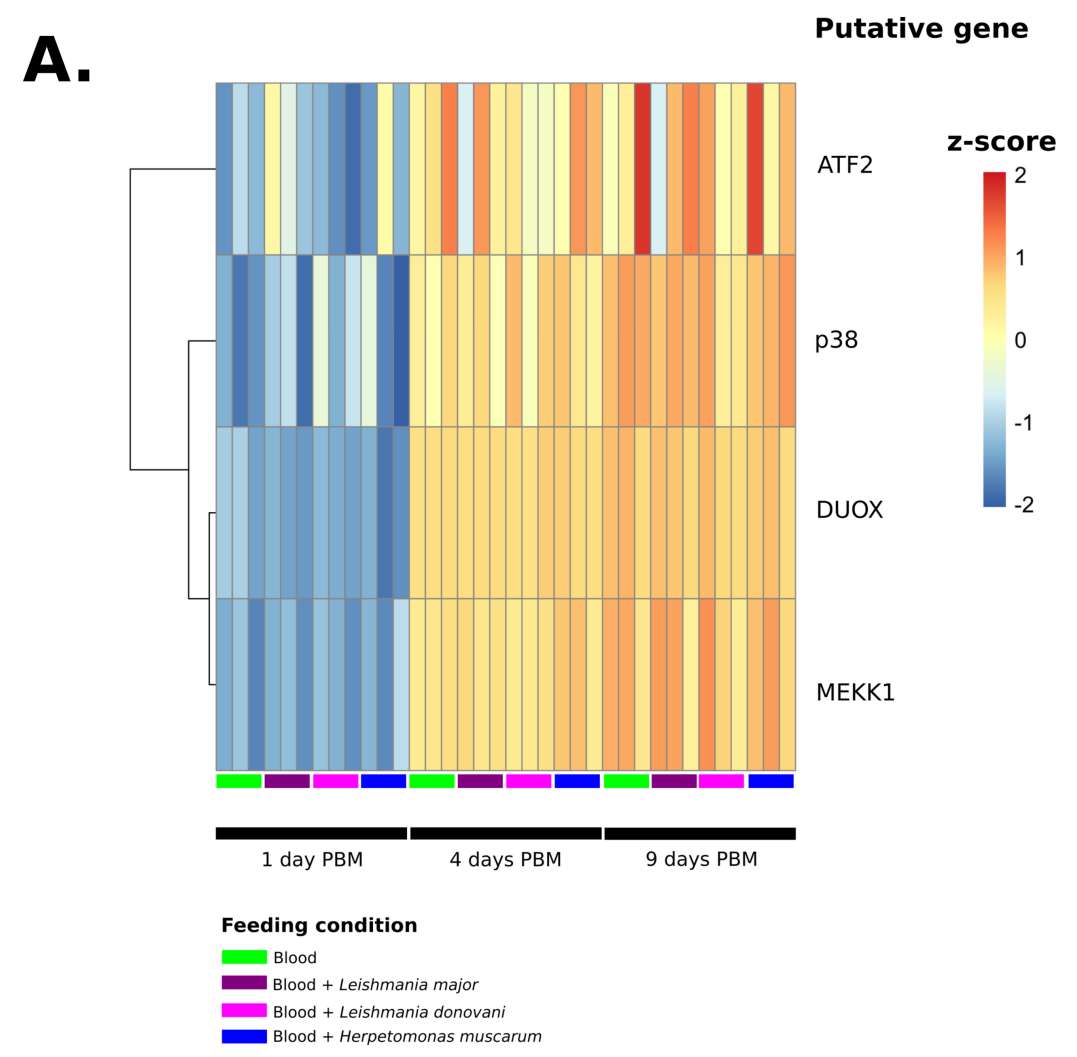

B.

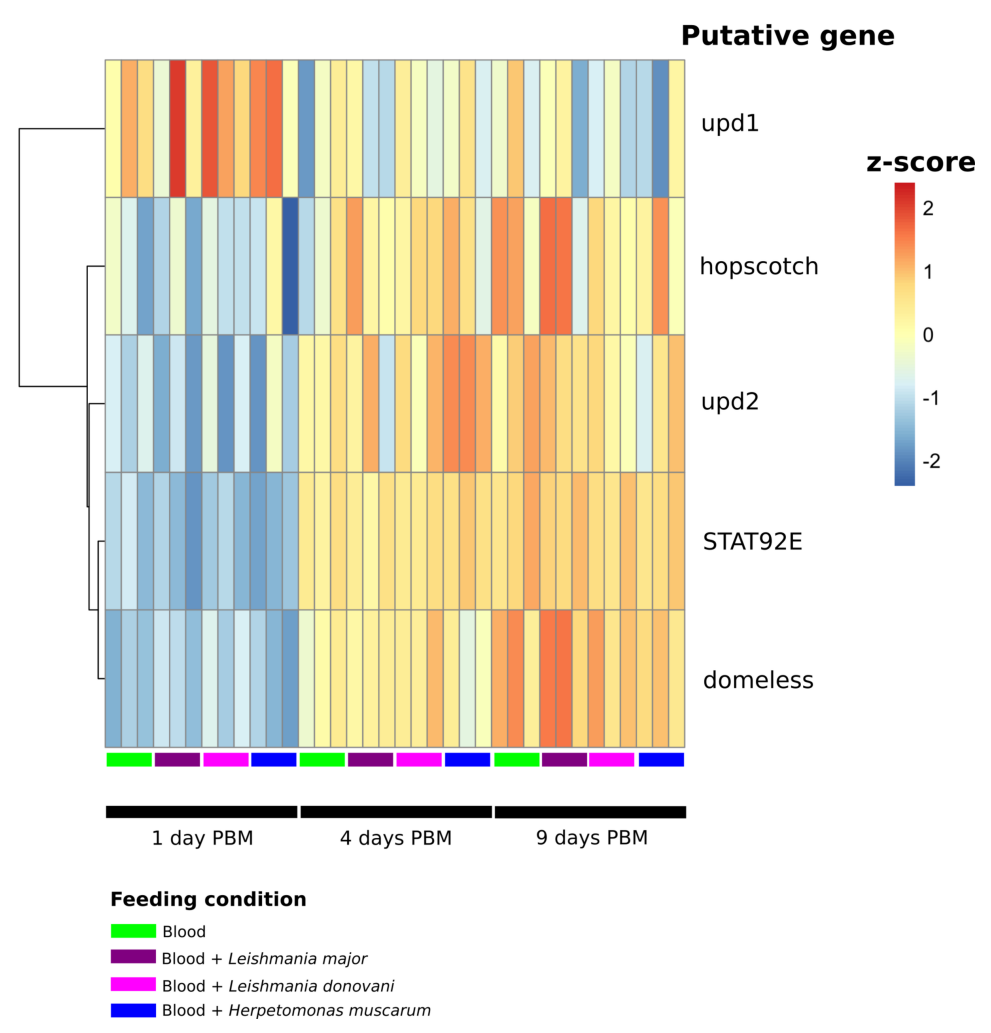

693 Figure 4 - Transcription of genes form the DUOX (A) and JAK-STAT signalling pathways in

694 P. papatasi across samples. Heatmaps of z-scores (based on log transformed, normalised

counts data) across samples. 


\section{Per2 - trend}

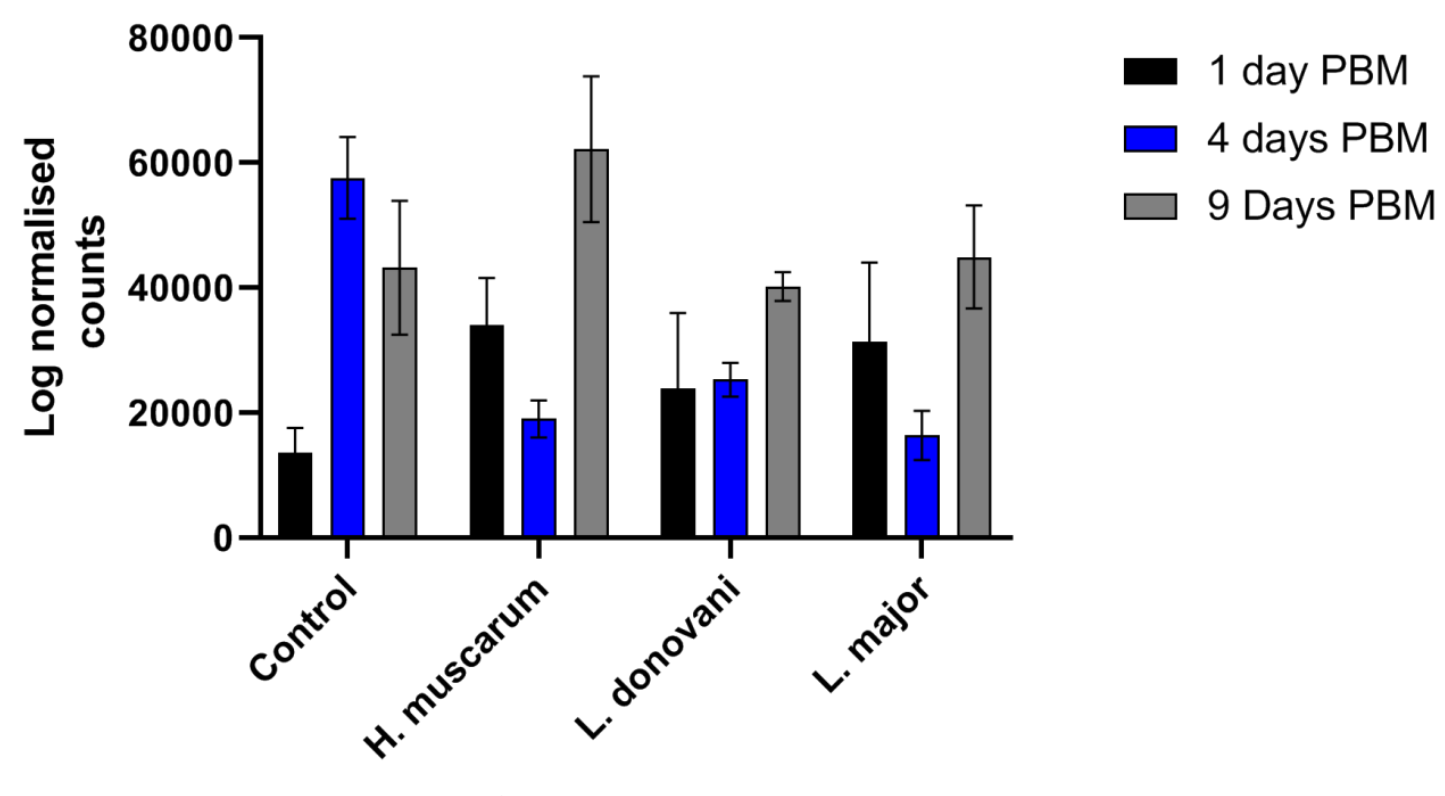

697

Infection condition

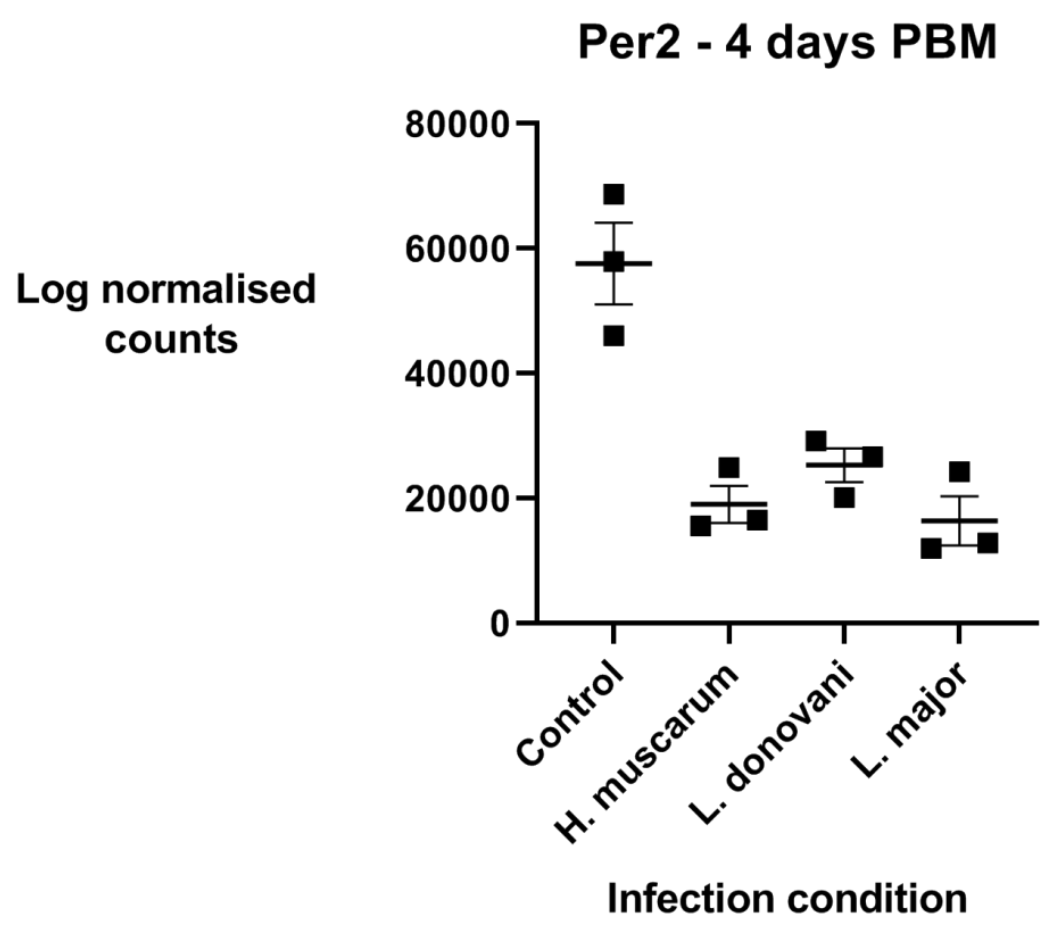

698

Figure S1 - Log normalised transcript counts for Peritrophin 2 (Per2) in P. papatasi

throughout infection. Error bars show the standard error of the mean. 
701 List of Supplementary Tables

702

703

Table S1 - Read mapping summaries. This table shows the read mapping information for

704

each sample e.g. number of reads, percentage of read mapped etc.

705

706

Table S2 - Transcripts associated with trypanosomatid presence in the blood meal. This

707

table shows the fold changes and differential regulation statistics (including p-values) for

708

transcripts whose abundance differed between trypanosomatid fed flies and blood-fed

709

control flies.

710

711 Table S3 - Transcripts associated with specific trypanosomatids in the blood meal. This

712 table shows the fold changes and differential regulation statistics (including p-values) for

713 transcripts whose abundance differed between trypanosomatid infections.

714

715

Table S4 - Transcripts significantly differentially regulated between 1 day and 4 days post

716

bloodmeal (blood-only) in P. papatasi.

717

718

Table S5 - Transcripts significantly differentially regulated between 4 day and 9 days post

bloodmeal (blood-only) in P. papatasi.

720

721 Table S6 - Transcripts of interest which are differentially regulated between 1 day and 4

722 days post bloodmeal (blood-only) in P. papatasi. This is a streamlined version of

723 supplementary table 4 showing transcripts of interest discussed in the text. 
724 Table S7 - Transcripts of interest which are differentially regulated between 4 days and 9

725 days post bloodmeal (blood-only) in P. papatasi. This is a streamlined version of

726 supplementary table 4 showing transcripts of interest discussed in the text.

727

728 Table S8 - Differential regulation statistics for transcripts of dipteran immune pathways of

729 interest (Toll, Imd, DUOX and JAK-STAT) across samples. ns - not significantly differentially 730 regulated.

731

732

733 List of supplementary data files

734 Annotation file for de novo assembled transcripts merged with Ppal1 annotation (.gtf)

735 Assembled transcript sequences (.fasta) 